\title{
Role of extracellular signal-regulated kinase (ERK)1/2 in multicellular resistance to docetaxel in MCF-7 cells
}

\author{
EUI KYONG JEONG, SU YEON LEE, HYUN MIN JEON, MIN KYUNG JU, \\ CHO HEE KIM and HO SUNG KANG \\ Department of Molecular Biology, College of Natural Sciences, Pusan National University, Pusan 609-735, Korea
}

Received February 1, 2010; Accepted April 22, 2010

DOI: 10.3892/ijo_00000714

\begin{abstract}
Cancer cells frequently fail to respond to chemotherapy due to acquisition of chemoresistance. Tumour cells are prone to die by necrosis when they are metabolically stressed by hypoxic and glucose depletion (OGD) due to insufficient vascularization, a common feature of solid tumours. Tumour necrosis indicates poor prognosis and emergence of drug resistance in cancer patients; however, its molecular mechanism remains unclear. In this study, we used multicellular tumour spheroids (MTS) as an in vitro tumour model to investigate the molecular mechanisms underlying necrosis-linked drug resistance. MCF-7 cells formed tight and spherical shape of spheroids and started to form the necrotic core at 8 days of culture. We found that docetaxel (DOC)induced apoptosis was gradually reduced during MCF-7 spheroid culture compared to that in monolayers and that more prominent resistance to DOC was observed when spheroids containing the necrotic core were treated. ERK1/2 and Akt appeared to be activated in MCF-7 spheroids with necrotic core, but not in 2D culture cells and in spheroids without necrotic core. DOC resistance in spheroids was reversed by inhibition of ERK1/2, but not of Akt, suggesting an important role for ERK1/2 in the DOC resistance in MCF-7 spheroids. These results provide new insight into the possible relation between necrosis-linked ERK1/2 activation and acquisition of multicellular resistance.
\end{abstract}

\section{Introduction}

Development of resistance to chemotherapy is frequently observed in malignant tumour cells $(1,2)$. Resistance to chemotherapy is complex and is acquired by several factors including increased drug efflux due to enhanced expression of ATP binding cassette transporter proteins, alterations in

Correspondence to: Dr Ho Sung Kang, Department of Molecular Biology, College of Natural Sciences, Pusan National University, Pusan 609-735, Korea

E-mail: hspkang@pusan.ac.kr

Key words: multicellular resistance, multicellular tumour spheroids, necrosis, ERK1/2, docetaxel drug activation/inactivation or drug targets, or inhibition of apoptotic pathways due to inactivation of the tumour suppressor p53 (1-3). Cancer cells also acquire resistance through activation of survival signaling pathways, such as the phosphatidylinositol-3 kinase (PI3K)/Akt pathway, the extracellular signal-regulated kinase (ERK) pathway, and the mammalian target of rapamycin (mTOR) pathway that play important roles in diverse cellular functions such as proliferation, differentiation, survival, and metabolism (4-9). The Ser/Thr kinase Akt/PKB is positively regulated by PI3K and negatively regulated by phosphatase PTEN and is frequently hyperactivated in human cancer and contributes to tumourigenesis $(5,6)$. Akt signaling has been shown to prevent apoptosis directly through phosphorylating $\mathrm{Bcl}-2$ family proteins or indirectly by regulating p53 and nuclear factor- $\mathrm{KB}(\mathrm{NF}-\kappa \mathrm{B})$ activity $(4,5)$. ERK1/2, a member of the mitogen-activated protein kinase (MAPK) family, is also hyperactivated due to constitutive activation of Ras and B-Raf, upstream of ERK1/2, in many human cancers and plays an essential role in tumour progression as well as drug resistance $(7,8)$. ERK1/2 inhibits apoptosis by preventing p53 activity and regulating Bcl-2 family protein and MDR1 gene expression $(8,10-12)$. The Ser/Thr kinase mTOR is stimulated by nutrient signals and various other stimuli through either the PI3K/PTEN/Akt pathway or the Ras/Raf/MEK/ERK pathway. It is also frequently hyperactivated in many types of cancer and could promote tumourigenesis, angiogenesis, and metastasis. mTOR confers survival advantage to tumour cells exposed to hypoxia and chemotherapeutic treatment via either of its two downstream targets, S6K or 4E-BP1/eIF4E $(9,13,14)$.

Studies of drug resistance are usually conducted on twodimensional (2D) monolayers. However, a wide discrepancy between in vitro $2 \mathrm{D}$ model and in vivo tumours is observed. For this reason, three-dimensional (3D) spheroid model system has been recently used for chemoresistance studies because it mimics the growth characteristics of in vivo tumours more closely than cancer cell line monolayers. When grown in spheroids, cancer cells develop additional resistance, referred to as 'multicellular resistance' (MCR), through contact resistance and resistance inherent in the spheroid structure (15-17). Although limited drug permeability has been suggested as a major cause of MCR found in spheroids, recent studies also showed that most, but not all, anticancer drugs could penetrate throughout the spheroids $(18,19)$. Pro-survival pathway such as NF-kB or mTOR, and 
P-glycoprotein and p27 expression have been implicated in emergence of drug resistance in spheroids $(14,20,21)$.

The development of hypoxia or anoxia regions is a general feature of solid tumours and prolonged hypoxia mediates formation of necrotic core regions (22-24). Tumour necrosis is concerned as a prognostic indicator and is linked to acquisition of resistance to various types of tumour therapy (25-27). Necrosis is characterized by the disruption of the cell membrane, leading to the release of cellular contents including HMGB1 and other cytokines that could stimulate tumour cell growth and progression, unlike apoptosis which retains membrane integrity (28-31). Although the molecular mechanism underlying contribution of hypoxia-mediated necrosis to drug resistance has been studied by many investigators, it remains still unclear.

In this study, we investigated the molecular mechanisms underlying necrosis-linked drug resistance using multicellular tumour spheroids (MTS), an in vitro tumour model. MTS closely resemble avascular regions of large solid tumours. MTS exhibit a proliferation gradient with an outer rim of proliferating cells that surrounds an inner shell of G0phase cells and a core region. The core regions lack nutrient and oxygen via insufficient supply that result in oxygen and nutrient gradients. These phenomena lead to necrotic cell death in the inner regions, forming the necrotic core $(15,16)$. Taxotere (docetaxel, DOC), one of taxanes that include two members paclitaxel and docetaxel, is a widely used antimicrotubule chemotherapeutic agent in the treatment of several solid tumours, including advanced breast cancer resistance (32). DOC stabilizes microtubule dynamics, promoting mitotic arrest and apoptotic cell death through induction of p53, activation of the jun $\mathrm{N}$-terminal kinase pathway, induction of p21 and p27 protein, and Bcl-2 phosphorylation and bcl-xl downregulation (32-36). In this study, we show that DOCinduced apoptosis is markedly reduced in spheroids containing necrotic core compared to those without necrotic core. After necrotic core formation, ERK1/2 is activated and inhibition of its activity increases the sensitivity to DOC. These findings suggest that soluble factors secreted from necrotic core may reduce sensitivity to DOC possibly through activating the ERK1/2 survival pathway.

\section{Materials and methods}

Two- and three-dimensional cell cultures. Human breast adenocarcinoma cells MCF-7, MDA-MB-231, and MDA-MB361 were obtained from ATCC (Manassas, VA, USA) and grown in DMEM (WelGENE, Daegu, Korea) supplemented with $10 \%(\mathrm{v} / \mathrm{v})$ heat-inactivated FBS and $1 \%(\mathrm{v} / \mathrm{v})$ penicillinstreptomycin in a $37^{\circ} \mathrm{C}$ humidified incubator with $5 \% \mathrm{CO}_{2}$. In $2 \mathrm{D}$ cell culture, cells were cultured as recommended by ATCC. For the initiation of 3D cell culture, MCF-7, MDAMB-231, and MDA-MB-361 cells were seeded at a density of 400 cells in $200 \mu 1$ medium into $1.2 \%$ agarose-precoated 96-well plates. After 3 days of culture, $100 \mu 1$ of medium was replaced with fresh medium every 2 days.

Drug treatments. DOC and LY294002 were purchased from Sigma (St. Louis, MO, USA) and U0126 was purchased from Calbiochem (San Diego, CA, USA). DOC, LY294002 and
U0126 were dissolved in dimethyl sulfoxide. The drugs and inhibitors were then divided into aliquots and stored at $-20^{\circ} \mathrm{C}$ until used. The stocks were freshly diluted in culture medium before experiment. In 2D culture, cells were pretreated with the inhibitors for $1 \mathrm{~h}$ and treated with $100 \mathrm{nM}$ DOC for $24 \mathrm{~h}$. In $3 \mathrm{D}$ culture, equal numbers of spheroids on each of the days indicated in the Figures were transferred to $1.2 \%$ agar-coated $60 \mathrm{~mm}$ dishes and pretreated with the inhibitors for $3 \mathrm{~h}$, considering the thickness of spheroids, and treated with $100 \mathrm{nM}$ DOC for $24 \mathrm{~h}$.

Hoechst 33258 (HO) and propidium iodide (PI) doublestaining. To determine the cell death mode, apoptosis or necrosis, HO and PI (Invitrogen, Carlsbad, CA, USA) double staining was performed. In $2 \mathrm{D}$ culture, cells were stained with $\mathrm{HO}(1 \mu \mathrm{g} / \mathrm{ml})$ and PI $(5 \mu \mathrm{g} / \mathrm{ml})$ for $15 \mathrm{~min}$ at $37^{\circ} \mathrm{C}$, and then dissociated with trypsin/EDTA (37). In 3D culture, spheroids were trypsinized and then stained. The dissociated cells were fixed by $3.7 \%$ paraformaldehyde and mounted using VectaMount (Vector Laboratories, Burlingame, CA, USA). For each sample, three visual fields were selected randomly using the AxioVision image analysis system (Carl Zeiss, Jena, Germany). The results were expressed as the percentages of apoptotic and necrotic cells.

Spheroids were collected, washed with PBS, fixed in $1 \%$ paraformaldehyde in PBS overnight at $4^{\circ} \mathrm{C}$, and dehydrated in a graded series of ethanol that were embedded in paraffin. From the paraffin blocks, $6 \mu \mathrm{m}$ sections were cut and mounted on glass slides, deparaffinized, rehydrated, and stained with $\mathrm{HO}$ and PI as described above.

Annexin V-FITC (AV)/PI staining and FACS analysis. To quantitate apoptosis, AV/PI staining was performed with the apoptosis detection kit I (BD Pharmingen, San Diego, CA, USA) following the manufacturer's instructions. Spheroids were washed twice in cold PBS, trypsinized, and resuspended in $1 \mathrm{X}$ binding buffer at a concentration of $1 \times 10^{5}$ cells $/ 100 \mu 1$. Five microliters of AV and PI each was added. Cells were gently vortexed and incubated for $15 \mathrm{~min}$ at room temperature in the dark. Then $400 \mu 1$ of the $1 \mathrm{X}$ binding buffer was added to each tube and then cells were analyzed by FACS. The results were expressed as the percentages of apoptotic and necrotic cells.

Immunoblotting. Protein lysates obtained from 2D and 3D culture cells were measured using the Bradford assay (BioRad, Richmond, CA, USA) and equal amounts of protein lysates were separated by sodium dodecyl sulfate-polyacrylamide gel electrophoresis on $12 \%$ gels, and electrotransferred to NC paper. The detection antibodies for phospho-serine 473 Akt, Akt, phospho-ERK1/2 and ERK1/2 were from Cell Signaling (Danvers, MA, USA). Anti-PARP (Santa Cruz Biotechnology, Santa Cruz, CA, USA) and anti- $\alpha$-tubulin (Biogenex, San Ramon, CA, USA) antibodies were also used. After incubation with peroxidase-conjugated secondary antibody protein expression was detected using ECL Western Blotting Detection Reagents (Amersham Biosciences, Buckinghamshire, UK).

Statistical analysis. Statistical analysis was performed using Minitab statistical software (Minitab Inc., State College, PA) 
A

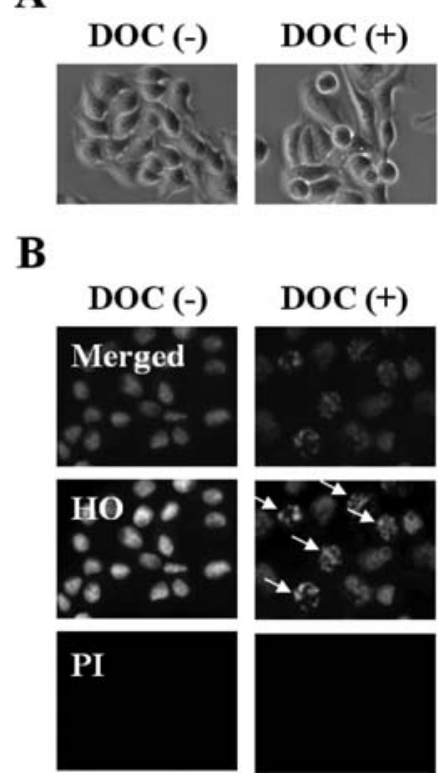

C

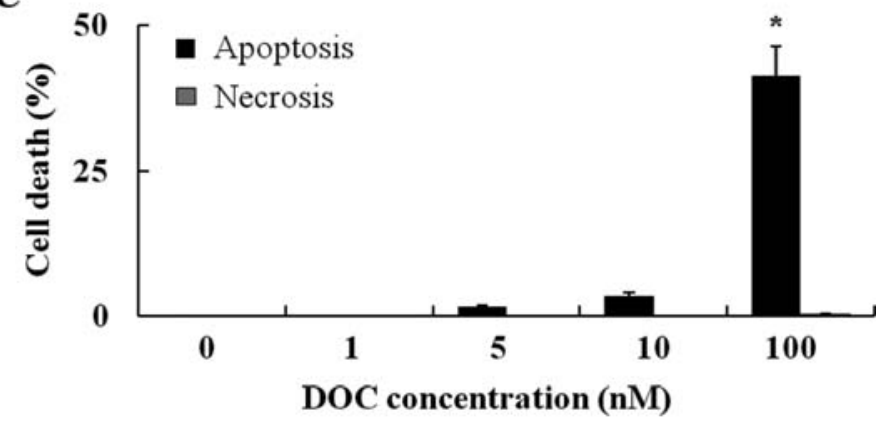

Figure 1. Formation of the necrotic core during MCF-7 MTS culture. (A) A growth curve of three breast cancer cell lines in MTS culture. MCF-7, MDA-MB-361, and MDA-MB-231 cells were seeded into 1.2\% agarosecoated 96-well plates at a density of 400 cells per well and cultured for up to 9 days (A). To calculate MTS size, diameters of 5 spheroids were measured every day (B). ${ }^{*} \mathrm{p}<0.05$. (C) After 7-11 days of MCF-7 MTS culture, MTS were dissociated into individual cells as described in 'Materials and methods' and stained with HO and PI and observed by fluorescence microscopy. Distinct PI positive nuclei, an indicator of necrosis, began to appear around 8 days of culture, indicating the necrotic core formation.

to compare data in different groups. $\mathrm{P}<0.05$ was considered statistically significant difference.

\section{Results and Discussion}

Formation of the necrotic core in MCF-7 spheroids. In this study, we tried to investigate the mechanism underlying necrosis-linked drug resistance using MTS, an in vitro tumour model (15-17). Three breast cancer cell lines MCF-7, MDAMB-231, and MDA-MB-361 were used to evaluate the formation of spheroids. The cells were seeded at 400 cells per well into 96-well plates pre-coated with $1.2 \%$ agar and the morphological features of spheroids were observed using light microscopy (Fig. 1A). When seeded in non-adhesive conditions, the cells aggregated and formed multicellular spheroids. While MDA-MB-231 cells generated only loose aggregates, MCF-7 and MDA-MB-361 cells formed tight aggregates. In particular, MCF-7 cells formed a compact spherical shape of spheroids, of which disintegration required extended trypsinization and physical strength. The differences in compact MTS formation between 3 cells are likely due to their differential expression of surface adhesion molecules, such as E-cadherin or N-cadherin. MCF-7 and MDA-MB-361 cells, but not MDA-MB-231 cells, are known to express Ecadherin on their surface (38). At 2 days after seeding, all spheroids were approximately 200-300 $\mu \mathrm{m}$ in diameter. MDA-MB-231 and MDA-MB-361 cells showed no significant change in spheroid size during 9 days of culture (Fig. 1B). In contrast, the size of MCF-7 spheroids increased gradually and reached approximately $700 \mu \mathrm{m}$ at 9 days (Fig. 1B).

Since MDA-MB-231 cells generated loose aggregates, we examined the formation of the necrotic core in MCF-7 and MDA-MB-361 spheroids. MCF-7 and MDA-MB-361 spheroids were collected and dissociated into single cells and stained with $\mathrm{HO}$ and PI to determine the cell death mode. DNA-binding dye $\mathrm{HO}$ penetrates the plasma membrane of all cells, whether they are damaged or not, causing a blue fluorescence of their nuclei and PI only penetrates cells with damaged membranes and leads to nuclear fluorescence. Thus, intact blue nuclei, condensed/fragmented blue nuclei, condensed/fragmented pink nuclei, and intact pink nuclei indicate viable, early apoptotic, late apoptotic (secondary necrotic), and necrotic cells, respectively. As shown in Fig. 1C, PI positive cells were detected beginning at 8 days in MCF-7 spheroids when the spheroids reached approximately 600$700 \mu \mathrm{m}$ in diameters. In contrast, no PI positive cells were observed in MDA-MB-361 cells, which generated less tight aggregates than MCF-7 cells (data not shown). We also observed the necrotic core formation at 8 days in MCF-7 spheroids, of which paraffin sections were stained with $\mathrm{HO}$ and PI (Fig. 3D). These results indicate that MCF-7 spheroids form the necrotic core after 8 days of culture. In MTS, oxygen diffusion-depleted zone is observed within 200 to $250 \mu \mathrm{m}$ from the rim and thus oxygen supply is limited in the core region, and central necrotic core is formed when the spheroids reached more than $500 \mu \mathrm{m}$ in diameters (39).

DOC-induced cell death in 2D culture. Then, we examined the cytotoxic effects of anti-cancer drug DOC. First, MCF-7 cells were treated with different concentrations of DOC (1-100 nM) for $12-48 \mathrm{~h}$ in $2 \mathrm{D}$ cell culture. Treatment of DOC caused a dose-dependent decrease in the cell viability, with prominent inhibitory effect on cell viability observed at a concentration of $100 \mathrm{nM}$. After $24 \mathrm{~h}$ exposure to $100 \mathrm{nM}$ DOC, we observed cell detachment and multinucleated giant cells due to mitotic arrest by light microscopy (Fig. 2A). In addition, MCF-7 cells treated with DOC at concentrations of $100 \mathrm{nM}$ for $24 \mathrm{~h}$ showed the characteristic features of apoptotic cell death, i.e. nuclear chromatin condensation and the appearance of typical apoptotic bodies (Fig. 2B and C).

Acquisition of prominent chemoresistance in MCF-7 spheroids after formation of central necrotic core. Next, we examined whether necrotic core formation influences DOC-induced apoptotic cell death in MCF-7 spheroids. DOC-induced apoptosis appeared to be gradually reduced during MCF-7 


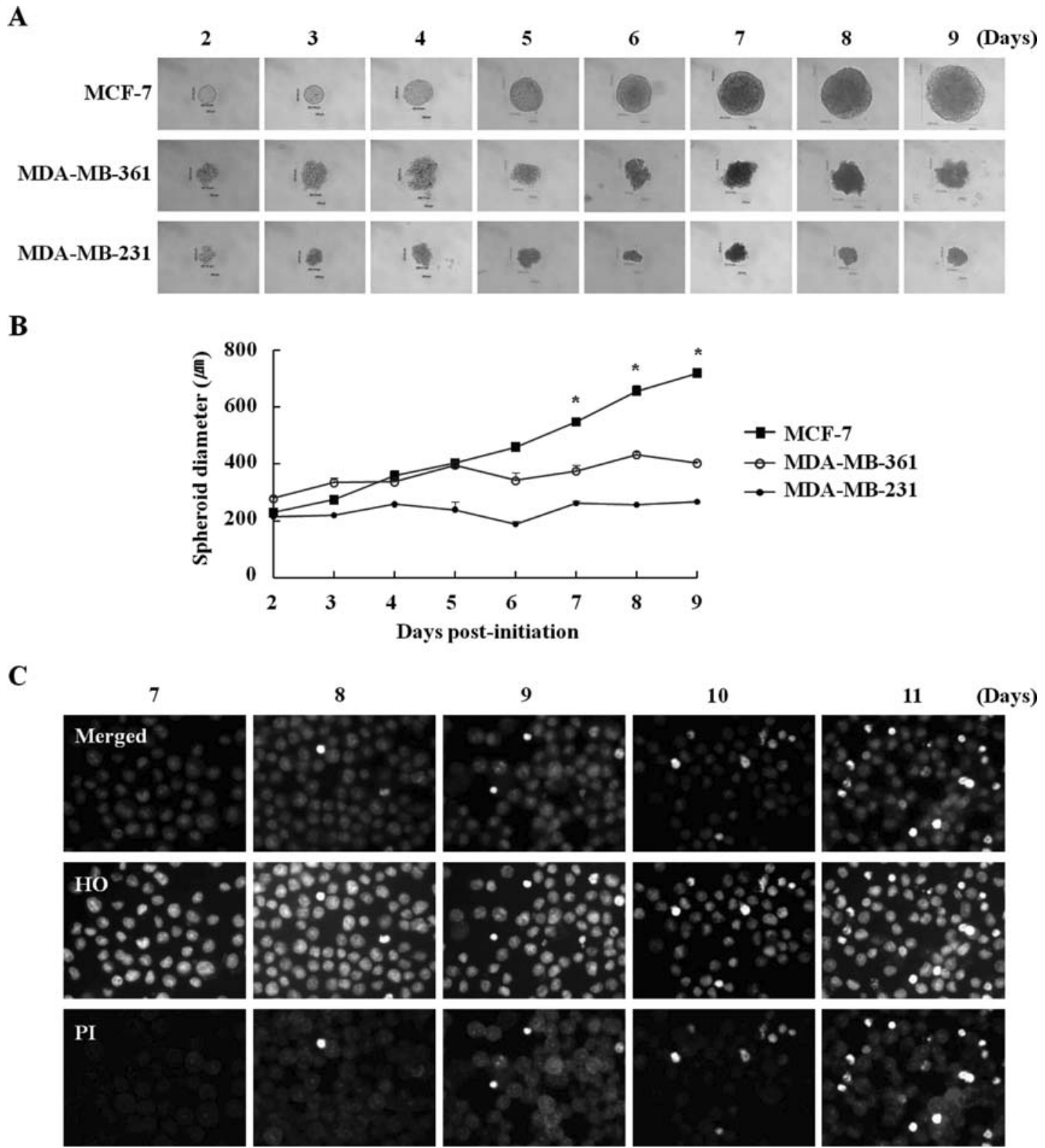

Figure 2. DOC-induced apoptotic cell death in MCF-7 monolayer. (A and B) MCF-7 cells were exposed to $100 \mathrm{nM}$ DOC for $24 \mathrm{~h}$ and observed under light microscopy (A, detached cells were observed following exposure to DOC) and stained with HO and PI and observed by fluorescence microscopy (B, arrow indicates apoptotic cells). (C) MCF-7 cells were treated with 1-100 nM DOC for $24 \mathrm{~h}$ and then stained with HO and PI and observed by fluorescence microscopy and apoptotic and necrotic cells were scored. Results (500-800 cells in each group) are expressed as the means \pm SEM from three independent experiments.

spheroid culture with increased percentage of necrotic cells compared to that in 2D cell cultures: DOC caused more than $40 \%$ of cells to undergo apoptosis in monolayers, but approximately $30 \%$ of cells to die by apoptosis at 7-8 days of MTS (Fig. 3A and B). Similar apoptotic resistance in multicellular spheroids has been reported by other investigators (15-17). Importantly, more prominent resistance to DOC was observed when treated to the spheroids containing necrotic cores: at day 9 of MTS, less than $10 \%$ of cells appeared to undergo apoptosis following exposure to DOC (Fig. 3A and B). Immunoblot analysis for PARP also showed that DOCinduced PARP cleavage was significantly reduced at 9 days of $3 \mathrm{D}$ culture, compared to that of 2D culture cells and 5 days of 3D culture cells (Fig. 3C). To confirm this reduction in chemosensitivity, we examined the apoptotic cell death in spheroids paraffin sections that were stained with $\mathrm{HO}$ and PI. DOC-induced apoptotic cells were observed at the peripheral rim, proliferating region at 6-7 days in 3D spheroids (Fig. 3D and E). Since DOC, as an anti-mitotic agent, leads to mitotic arrest and apoptosis only in proliferating cells, DOC-induced apoptotic cells are likely detected only at the peripheral rim. As expectedly, DOC-induced apoptotic cells appeared to be significantly diminished at 9 days in 3D spheroids that had the necrotic core (Fig. 3D). These results suggest that emergence of central necrosis may play a crucial role(s) in development of resistance to DOC. Although limited drug permeability has been suggested as a major cause of MCR found in spheroids, recent studies also showed that most, but 
A

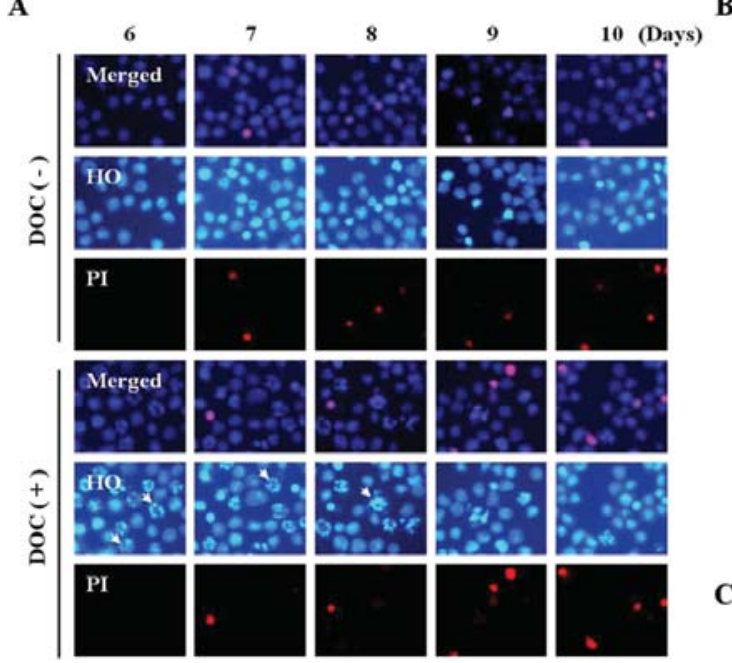

D

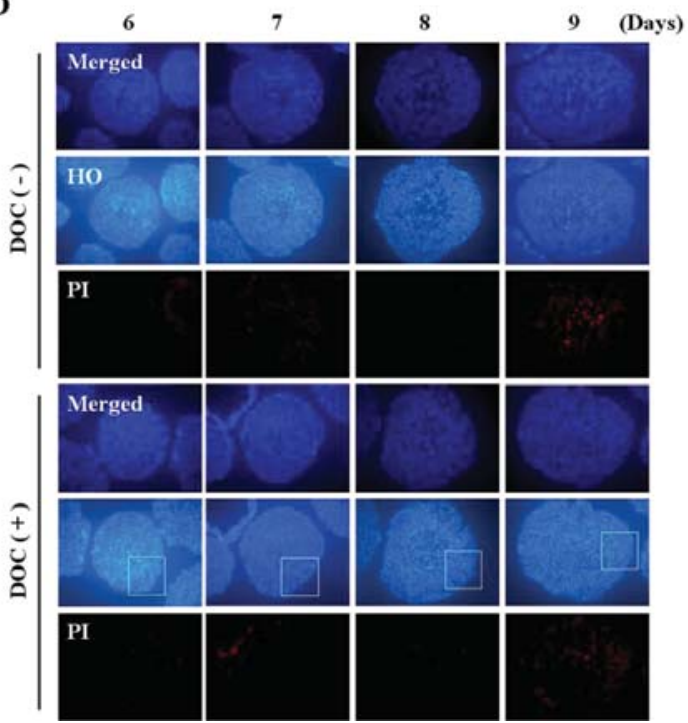

$\mathbf{E}$
B

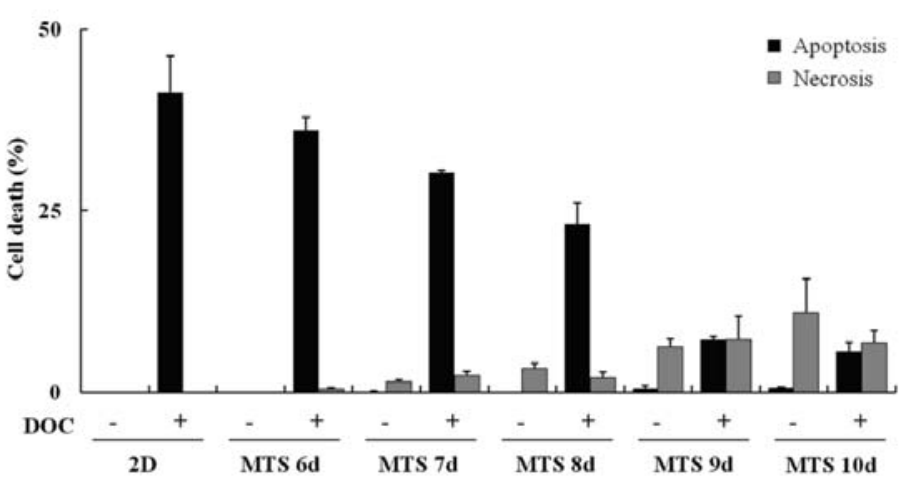

C

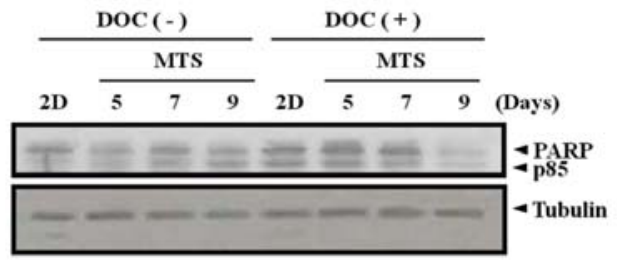

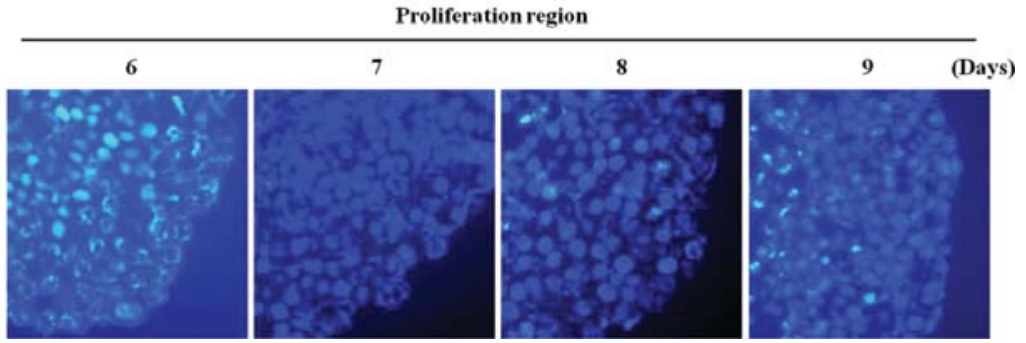

Figure 3. Acquisition of resistance to DOC in MCF-7 spheroids. (A and B) MCF-7 cells were seeded into $1.2 \%$ agarose-coated 96-well plates at a density of 400 cells per well and cultured for up to 10 days and exposed to $100 \mathrm{nM} \mathrm{DOC}$ for $24 \mathrm{~h}$. MTS were dissociated into individual cells as described in 'Materials and methods' and stained with HO and PI and observed by fluorescence microscopy (A). Apoptotic and necrotic cells were scored. Results (500-800 cells in each group) are expressed as the means \pm SEM from three independent experiments (B). (C) MCF-7 cells were seeded into 1.2\% agarose-coated 96-well plates at a density of 400 cells per well and cultured for up to 9 days and exposed to $100 \mathrm{nM} \mathrm{DOC}$ for $24 \mathrm{~h}$ and the resulting cellular proteins were analyzed by Western blot using specific antibodies for PARP. $\alpha$-tubulin was used as a loading control. (D and E) MCF-7 cells were seeded into $1.2 \%$ agarose-coated 96-well plates at a density of 400 cells per well and cultured for up to 9 days and exposed to $100 \mathrm{nM}$ DOC for $24 \mathrm{~h}$ and the paraffin sections were stained with $\mathrm{HO}$ and PI (D). The large images in the panel $\mathrm{E}$ are the enlargement of the box in the panel D.

not all, anticancer drugs could penetrate throughout the spheroids $(18,19)$. Thus, the apoptotic resistance to DOC at 9 days is not likely due to its limited diffusion.

Activation of the Akt and ERK survival pathways in MCF-7 spheroids with necrotic core. Development of resistance to DOC has been reported to involve an activation of the survival signaling pathways, such as the Akt and ERK pathways $(35,36)$. To investigate whether these signaling pathways are activated in MCF-7 spheroids, cellular proteins obtained from 2D culture and spheroids were analyzed by Western blotting using antibodies to phospho-Akt and phospho-ERK. As shown in Fig. 4, increased phosphorylation in Akt and ERK proteins was detected at 9 day MTS with the necrotic core compared to $2 \mathrm{D}$ culture and spheroids without necrotic core, indicating that Akt and ERK activation is closely linked to the necrotic core formation in MTS.

Inhibition of ERK activity overcomes resistance to DOC in MCF-7 spheroids. To determine whether Akt and ERK activation contributes to necrosis-linked DOC resistance, we examined the effects of inhibition of Akt or ERK on resistance to DOC in MCF-7 spheroids. LY294002 and U0126 alone did not exert the cytotoxic effects in the MTS. While pretreatment of LY294002 and U0126 exerts no significant effects on DOC-induced apoptosis in 5 day spheroids, U0126, but not LY294002, markedly suppressed the acquired cellular resistance to DOC observed in 9 day MTS (Fig. 5A and B). $\mathrm{HO}$ and PI staining of paraffin sections from 9-day MCF-7 spheroids also showed that U0126 markedly sensitized the 


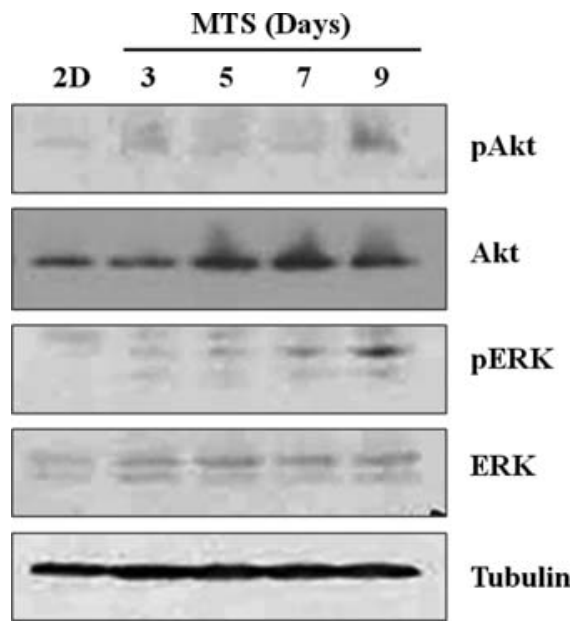

Figure 4. Activation of Akt and ERK1/2 in MCF-7 spheroids after necrotic core formation. MCF-7 cells were seeded into $1.2 \%$ agarose-coated 96-well plates at a density of 400 cells per well and cultured for up to 9 days and the resulting cellular proteins were analyzed by Western blot using specific antibodies for Akt, phospho-Akt, ERK and phospho-ERK. $\alpha$-tubulin was used as a loading control. cells to DOC-induced apoptosis observed in the outer rim (Fig. 5C and D). Thus, ERK signaling may contribute to necrosis-linked DOC resistance in MCF-7 spheroids containing a necrotic core.

We showed that ERK activation in MTS may be linked to necrosis. ERK may be activated by the molecules (e.g. HMGB1) released by necrosis or OGD stress. ERK1/2 is known to inhibit apoptosis by preventing p53 activity and regulating Bcl-2 family protein and MDR1 gene expression $(8,10-12)$. Since the ERK pathway could activate mTOR whose inhibition prevents acquired apoptotic resistance in spheroids (14), ERK-mTOR pathway may act as a mediator of the acquired resistance in spheroids. Thus, it will be interesting to examine the molecular target of ERK1/2 responsible for the acquired apoptotic resistance.

These results provide new insight into the role of ERK on chemoresistance using 3D model system. Many investigators have proposed that formation of a necrotic core in solid tumours may contribute to drug resistance. Especially, hypoxia is known to frequently occur in human tumours due to lack of oxygen supply and to lead to intratumoural necrotic

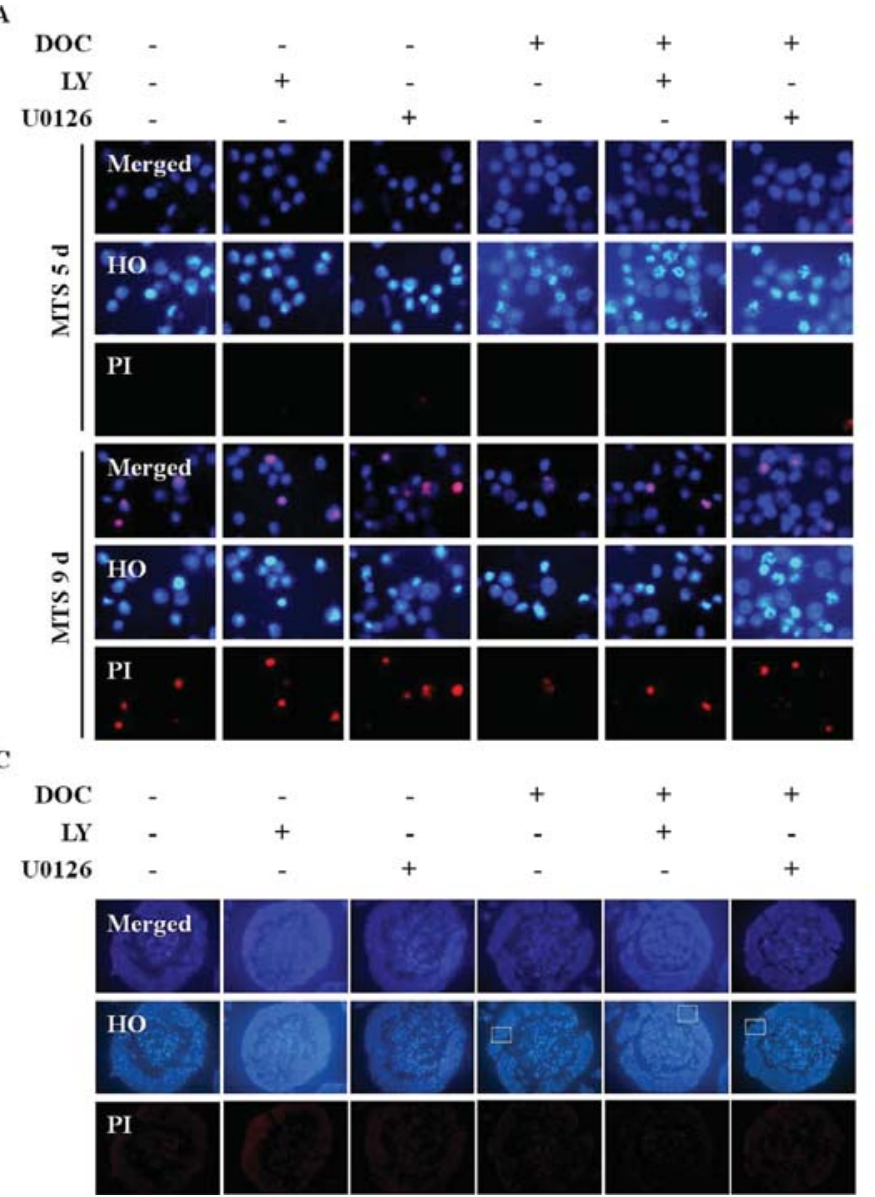

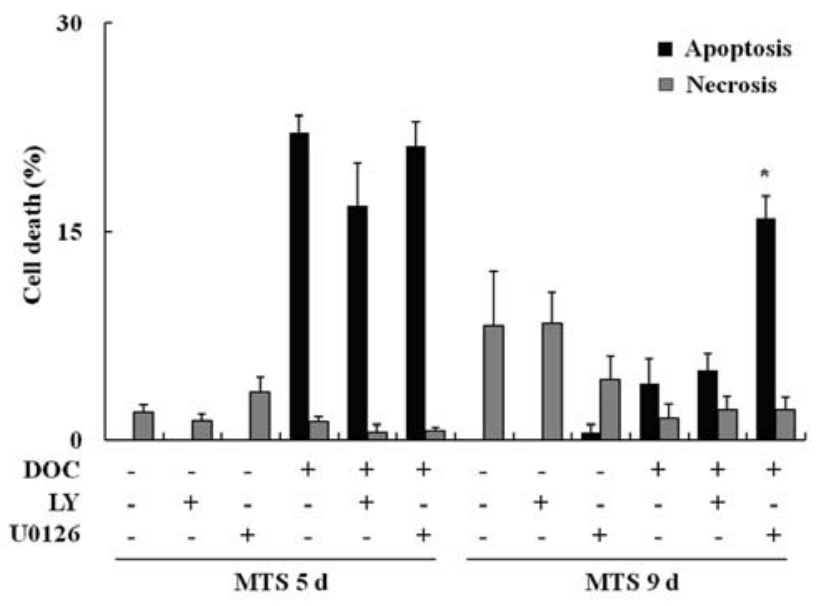

D

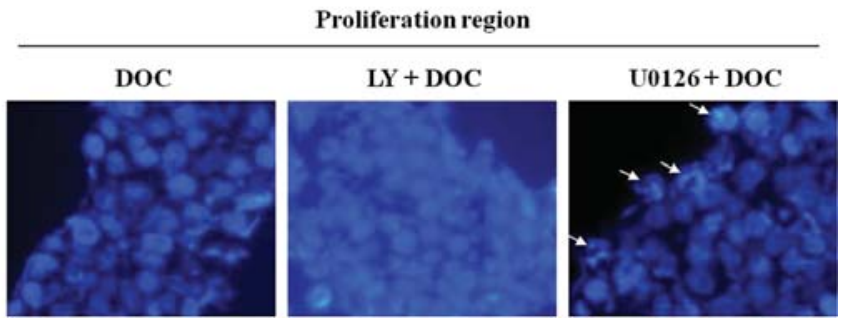

Figure 5. Inhibition of ERK1/2 abolishes MCF-7 spheroid resistance to DOC. (A and B) MCF-7 cells were seeded into 1.2\% agarose-coated 96-well plates at a density of 400 cells per well and cultured for 5 and 9 days and were pretreated for $3 \mathrm{~h}$ with either $20 \mu \mathrm{M} \mathrm{LY} 294002$ or $20 \mu \mathrm{M} \mathrm{U} 0126$ and then exposed to $100 \mathrm{nM}$ DOC for $24 \mathrm{~h}$. MTS were dissociated into individual cells as described in 'Materials and methods' and stained with HO and PI and observed by fluorescence microscopy (A, arrow indicates apoptotic cells). Apoptotic and necrotic cells were scored. Results (500-800 cells in each group) are expressed as the means \pm SEM from three independent experiments (B). " $\mathrm{p}<0.05$. (C and D) MCF-7 cells were seeded into $1.2 \%$ agarose-coated 96 -well plates at a density of 400 cells per well and cultured for 9 days and were pretreated for $3 \mathrm{~h}$ with either $20 \mu \mathrm{M} \mathrm{LY} 294002$ or $20 \mu \mathrm{M} \mathrm{U} 0126$ and then exposed to $100 \mathrm{nM}$ DOC for $24 \mathrm{~h}$. The paraffin sections were stained with HO and PI (C). The large images in the panel D are the enlargement of the box in the panel C. Arrows indicate apoptotic cells. 
cell death and drug resistance. Our results may explain the mechanism underlying hypoxia-induced necrosis in chemoresistance. Further studies on the role of necrosis in resistance are required to advance the understanding of the mechanism of resistance in solid tumours.

\section{Acknowledgements}

This study was supported by a grant from the National R\&D Program for Cancer Control, Ministry of Health and Welfare, Republic of Korea (0620010), and the National Research Foundation of Korea (NRF) grant funded by the Korea Government (MEST) (2009-0059210).

\section{References}

1. Raguz S and Yague E: Resistance to chemotherapy: new treatments and novel insights into an old problem. Br J Cancer 99: 387-391, 2008.

2. Wilson TR, Longley DB and Johnston PG: Chemoresistance in solid tumours. Ann Oncol 17 (Suppl. 10): S315-S324, 2006.

3. Meads MB, Gatenby RA and Dalton WS: Environment-mediated drug resistance: a major contributor to minimal residual disease. Nat Rev Cancer 9: 665-674, 2009.

4. Yap TA, Carden CP and Kaye SB: Beyond chemotherapy: targeted therapies in ovarian cancer. Nat Rev Cancer 9: 167-181, 2009.

5. Vivanco I and Sawyers CL: The phosphatidylinositol 3-Kinase AKT pathway in human cancer. Nat Rev Cancer 2: 489-501, 2002.

6. Tokunaga E, Oki E, Egashira A, Sadanaga N, Morita M, Kakeji Y and Maehara Y: Deregulation of the Akt pathway in human cancer. Curr Cancer Drug Targets 8: 27-36, 2008.

7. McCubrey JA, Steelman LS, Chappell WH, Abrams SL, Wong EW, Chang F, Lehmann B, Terrian DM, Milella M, Tafuri A, Stivala F, Libra M, Basecke J, Evangelisti C, Martelli AM and Franklin RA: Roles of the Raf/MEK/ERK pathway in cell growth, malignant transformation and drug resistance. Biochim Biophys Acta 1773: 1263-1284, 2007.

8. Balmanno $\mathrm{K}$ and Cook SJ: Tumour cell survival signalling by the ERK1/2 pathway. Cell Death Differ 16: 368-377, 2009.

9. Jiang BH and Liu LZ: Role of mTOR in anticancer drug resistance: perspectives for improved drug treatment. Drug Resist Updat 11: 63-76, 2008.

10. Katayama K, Yoshioka S, Tsukahara S, Mitsuhashi J and Sugimoto $\mathrm{Y}$ : Inhibition of the mitogen-activated protein kinase pathway results in the down-regulation of P-glycoprotein. Mol Cancer Ther 6: 2092-2102, 2007.

11. Kisucka J, Barancik M, Bohacova V and Breier A: Reversal effect of specific inhibitors of extracellular-signal regulated protein kinase pathway on P-glycoprotein mediated vincristine resistance of L1210 cells. Gen Physiol Biophys 20: 439-444, 2001

12. McCubrey JA, Abrams SL, Ligresti G, Misaghian N, Wong EW, Steelman LS, Basecke J, Troppmair J, Libra M, Nicoletti F, Molton S, McMahon M, Evangelisti C and Martelli AM: Involvement of p53 and Raf/MEK/ERK pathways in hematopoietic drug resistance. Leukemia 22: 2080-2090, 2008.

13. Wouters BG and Koritzinsky M: Hypoxia signalling through mTOR and the unfolded protein response in cancer. Nat Rey Cancer 8: 851-864, 2008.

14. Barbone D, Yang TM, Morgan JR, Gaudino G and Broaddus VC: Mammalian target of rapamycin contributes to the acquired apoptotic resistance of human mesothelioma multicellular spheroids. J Biol Chem 283: 13021-13030, 2008.

15. Friedrich J, Seidel C, Ebner R and Kunz-Schughart LA: Spheroid-based drug screen: considerations and practical approach. Nat Protoc 4: 309-324, 2009.

16. Gottfried E, Kunz-Schughart LA, Andreesen R and Kreutz M: Brave little world: spheroids as an in vitro model to study tumour-immune-cell interactions. Cell Cycle 5: 691-695, 2006.

17. Horning JL, Sahoo SK, Vijayaraghavalu S, Dimitrijevic S, Vasir JK, Jain TK, Panda AK and Labhasetwar V: 3-D tumour model for in vitro evaluation of anticancer drugs. Mol Pharm 5: 849-862, 2008.
18. Desoize B and Jardillier J: Multicellular resistance: a paradigm for clinical resistance? Crit Rev Oncol Hematol 36: 193-207, 2000.

19. West GW, Weichselbaum R and Little JB: Limited penetration of methotrexate into human osteosarcoma spheroids as a proposed model for solid tumour resistance to adjuvant chemotherapy. Cancer Res 40: 3665-3668, 1980.

20. Xing H, Wang S, Weng D, Chen G, Yang X, Zhou J, Xu G, Lu Y and Ma D: Knock-down of P-glycoprotein reverses taxol resistance in ovarian cancer multicellular spheroids. Oncol Rep 17: 117-122, 2007.

21. Gallardo-Perez JC, Espinosa M, Ceballos-Cancino G, Daniel A, Rodriguez-Enriquez S, Aviles A, Moreno-Sanchez R, Melendez-Zajgla $\mathrm{J}$ and Maldonado $\mathrm{V}$ : NF-kappa $\mathrm{B}$ is required for the development of tumour spheroids. J Cell Biochem 108: 169-180, 2009.

22. Gatenby RA and Gillies RJ: Why do cancers have high aerobic glycolysis? Nat Rev Cancer 4: 891-899, 2004.

23. Bristow RG and Hill RP: Hypoxia and metabolism. Hypoxia, DNA repair and genetic instability. Nat Rev Cancer 8: 180-192, 2008.

24. Brown JM: Tumour microenvironment and the response to anticancer therapy. Cancer Biol Ther 1: 453-458, 2002.

25. Edwards JG, Swinson DE, Jones JL, Muller S, Waller DA and O'Byrne KJ: Tumour necrosis correlates with angiogenesis and is a predictor of poor prognosis in malignant mesothelioma. Chest 124: 1916-1923, 2003.

26. Langner C, Hutterer G, Chromecki T, Leibl S, Rehak P and Zigeuner R: Tumour necrosis as prognostic indicator in transitional cell carcinoma of the upper urinary tract. J Urol 176: 910-914, 2006.

27. Leibovitch I, Lev R, Mor Y, Golomb J, Dotan ZA and Ramon J: Extensive necrosis in renal cell carcinoma specimens: potential clinical and prognostic implications. Isr Med Assoc J 3: 563-565, 2001.

28. Vakkila $\mathrm{J}$ and Lotze MT: Inflammation and necrosis promote tumour growth. Nat Rev Immunol 4: 641-648, 2004.

29. Lotze MT and Tracey KJ: High-mobility group box 1 protein (HMGB1): nuclear weapon in the immune arsenal. Nat Rev Immunol 5: 331-342, 2005.

30. Brown GD: Sensing necrosis with Mincle. Nat Immunol 9: 1099-1100, 2008

31. Kepp O, Tesniere A, Schlemmer F, Michaud M, Senovilla L, Zitvogel L and Kroemer G: Immunogenic cell death modalities and their impact on cancer treatment. Apoptosis 14: 364-375, 2009.

32. McGrogan BT, Gilmartin B, Carney DN and McCann A: Taxanes, microtubules and chemoresistant breast cancer. Biochim Biophys Acta 1785: 96-132, 2008.

33. Hernandez-Vargas H, Palacios J and Moreno-Bueno G: Molecular profiling of docetaxel cytotoxicity in breast cancer cells: uncoupling of aberrant mitosis and apoptosis. Oncogene 26: 2902-2913, 2007.

34. Honma K, Iwao-Koizumi K, Takeshita F, Yamamoto Y, Yoshida T, Nishio K, Nagahara S, Kato K and Ochiya T: RPN2 gene confers docetaxel resistance in breast cancer. Nat Med 14: 939-948, 2008.

35. Jiang CC, Yang F, Thorne RF, Zhu BK, Hersey P and Zhang XD: Human melanoma cells under endoplasmic reticulum stress acquire resistance to microtubule-targeting drugs through XBP-1mediated activation of Akt. Neoplasia 11: 436-447, 2009.

36. Navolanic PM, Lee JT and McCubrey JA: Docetaxel cytotoxicity is enhanced by inhibition of the Raf/MEK/ERK signal transduction pathway. Cancer Biol Ther 2: 677-678, 2003.

37. Kim CH, Han SI, Lee SY, Youk HS, Moon JY, Duong HQ, Park MJ, Joo YM, Park HG, Kim YJ, Yoo MA, Lim SC and Kang HS: Protein kinase C-ERK1/2 signal pathway switches glucose depletion-induced necrosis to apoptosis by regulating superoxide dismutases and suppressing reactive oxygen species production in A549 lung cancer cells. J Cell Physiol 211: 371-385, 2007.

38. Ivascu A and Kubbies M: Diversity of cell-mediated adhesions in breast cancer spheroids. Int J Oncol 31: 1403-1413, 2007.

39. Mueller-Klieser WF and Sutherland RM: Influence of convection in the growth medium on oxygen tensions in multicellular tumour spheroids. Cancer Res 42: 237-242, 1982. 